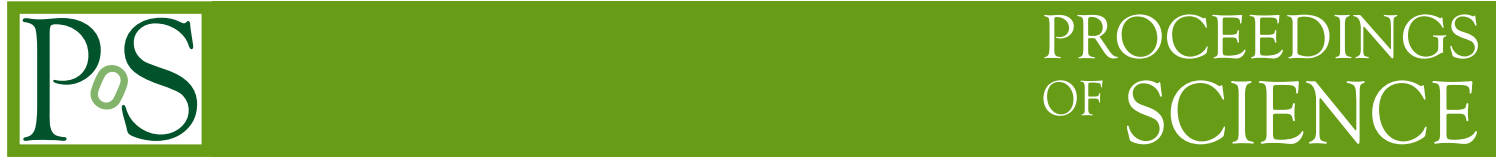

\title{
Progress in Lattice QCD at non-zero temperature and QGP
}

\section{Peter Petreczky ${ }^{* \dagger}$}

Physics Department, Brookhaven National Laboratory, Upton NY 11973

E-mail: petreczk@bnl.gov

I review recent progress in lattice $\mathrm{QCD}$ at non-zero temperature whith emphasis on the calculations of equation and the deconfinement as well as the chiral aspects of the QCD transition at non-zero temperature. I also briefly discuss meson correlation functions at high temperatures.

Light Cone 2010 - LC2010

June 14-18, 2010

Valencia, Spain

*Speaker.

${ }^{\dagger}$ This work has been supported by contract DE-AC02-98CH10886 with the U.S. Department of Energy. The numerical calculations have been performed using QCDOC superomputers of USQCD Collaboration and RIKEN-BNL Research Center, the clusters at FNAL, as well as the BlueGene/L at the New York Center for Computational Sciences (NYCCS). 


\section{Introduction}

It is expected that strongly interacting matter undergoes a transition in some temperature interval from hadron gas to deconfined state also called the quark gluon plasma (QGP) [1]. Creating deconfined medium in a laboratory is the subject of the large experimental program at RHIC [2] and is going to be the goal of the future heavy-ion program at LHC [3]. Attempts to study QCD thermodynamics on the lattice go back to the early 80's when lattice calculations in $S U(2)$ gauge theory provided the first rigorous theoretical evidence for deconfinement $[4,5,6]$. The problem of calculating thermodynamic observables in pure gluonic theory was solved in 1996 [7], while calculation involving dynamical quarks were limited to large quark masses and had no control over discretization errors $[8,9,10]$ (see Refs. [11, 12] for reviews). Lattice calculations of QCD thermodynamics with light dynamical quarks remained challenging until recently. During the past 5 years calculations with light $u, d$ quarks have been performed using improved staggered fermion actions [13, 14, 15, 16, 17, 18, 19, 20, 21, 22, 23, 24, 25, 26] (see Refs. [27, 28].)

To get reliable predictions from lattice QCD the lattice spacing $a$ should be sufficiently small relative to the typical QCD scale, i.e. $\Lambda_{Q C D} a \ll 1$. For staggered fermions, which are used for calculations at non-zero temperature, discretization errors go like $\mathscr{O}\left(\left(a \Lambda_{Q C D}\right)^{2}\right)$ but discretization errors due to flavor symmetry breaking turn out to be numerically quite large. To reduce these errors one has to use improved staggered fermion actions with so-called fat links [30]. At high temperature the dominant discretization errors go like $(a T)^{2}$ and therefore could be very large. Thus it is mandatory to use improved discretization schemes, which improve the quark dispersion relation and eliminate these discretization errors. Lattice fermion actions used in numerical calculations typically implement some version of fat links as well as improvement of quark dispersion relation and are referred to as $p 4$, asqtad, HISQ and stout. In lattice calculations the temperature is varied by varying the lattice spacing at fixed value of the temporal extent $N_{\tau}$. The temperature $T$ is related to lattice spacing and temporal extent, $T=1 /\left(N_{\tau} a\right)$. Therefore taking the continuum limit corresponds to $N_{\tau} \rightarrow \infty$ at the fixed physical volume. For the same reason discretization errors in the hadronic phase could be large when the temperature $T$ is small.

In this paper I discuss lattice QCD calculation of the transition temperature, the equation of state as well as different spatial and temporal correlation functions.

\section{Equation of State}

The equation of state has been calculated with $p 4$ and asqtad action on lattices with temporal extent $N_{\tau}=4,6$ and $8[17,18,20]$. In these calculations the strange quark mass was fixed to its physical value, while the light $(u, d)$ quark masses 10 times smaller than the strange quark mass have been used. These correspond to pion masses of $(220-260) \mathrm{MeV}$. The calculation of thermodynamic observables proceeds through the calculation of the trace of the energy momentum tensor $\varepsilon-3 p$ also known as trace anomaly or interaction measure. This is due to the fact that this quantity can be expressed in terms of expectation values of local gluonic and fermionic operators. The explicit expression for $\varepsilon-3 p$ in terms of these operators for $p 4$ and asqtad actions can be found in Ref. [20]. Different thermodynamic observables can be obtained from the interaction 
measure through integration. The pressure can be written as

$$
\frac{p(T)}{T^{4}}-\frac{p\left(T_{0}\right)}{T_{0}^{4}}=\int_{T_{0}}^{T} \frac{d T^{\prime}}{T^{\prime 5}}(\varepsilon-3 p) .
$$

The lower integration limit $T_{0}$ is chosen such that the pressure is exponentially small there. Furthermore, the entropy density can be written as $s=(\varepsilon+p) / T$. Since the interaction measure is the basic thermodynamic observable in the lattice calculations it is worth discussing its properties more in detail. In Fig. 1 I show the interaction measure for $p 4$ and asqtad actions for two different lattice spacings corresponding to $N_{\tau}=6$ and 8 . In the high temperature region, $T>250 \mathrm{MeV}$ results obtained with two different lattice spacings and two different actions agree quite well with each other. Furthermore, recent calculations with HISQ actions also give results for the trace anomaly which are consistent with these [24]. Discretization errors are visible in the temperature region, where $\varepsilon-3 p$ is close to its maximum as well as in the low temperature region. At low temperatures the lattice data have been compared with the hadron resonance gas (HRG). As one can see the lattice data fall below the resonance gas value. This is partly due to the fact that the light quark masses are still about two times larger than the physical value as well as to discretization errors. The $\mathscr{O}\left(\left(a \Lambda_{Q C D}\right)^{2}\right)$ discretization errors in the hadron spectrum are suppressed at high temperatures as the lattice spacing $a$ is small there. Also hadrons are not the relevant degrees of freedom in this temperature region. But at small temperatures, where hadrons are the relevant degrees of freedom, these discretization effects are significant. It turns out, however, that the HRG model that takes into account the quark mass dependence and discretization errors in the hadron spectrum can describe the lattice data quite well [31]. The large discretization errors in the low temperature region is the reason for the discrepancies with the stout results at temperatures $T<200 \mathrm{MeV}$ [23]. The lattice results for the trace anomaly obtained with stout action are also different in the high temperature limit [23]. Namely, $(\varepsilon-3 p) / T^{4}$ calculatied with stout action is about $50 \%$ smaller in the high temperature region. To resolve this problem calculation at finer lattice spacing are needed.
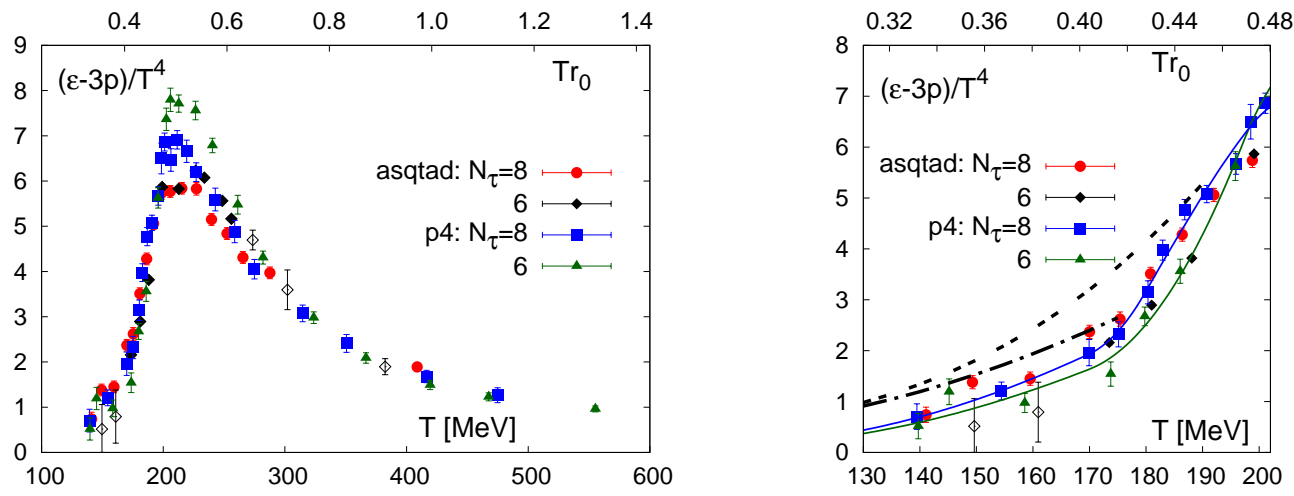

Figure 1: The interaction measure calculated with $p 4$ and asqtad actions in entire temperature range (left) and at low temperatures (right) from Ref. [20]. The dashed and dashed-dotted lines are the prediction of hadron resonance gas (HRG) with all resonances included up $2.5 \mathrm{GeV}$ (dashed) and $1.5 \mathrm{GeV}$ (dashed-dotted), respectively.

The pressure, the energy density and the entropy density are shown in Fig. 2. The energy density shows a rapid rise in the temperature region $(185-195) \mathrm{MeV}$ and quickly approaches 
about $90 \%$ of the ideal gas value. The pressure rises less rapidly but at the highest temperature it is also only about $15 \%$ below the ideal gas value. In the previous calculations with the $p 4$ action it was found that the pressure and energy density are below the ideal gas value by about $25 \%$ at high temperatures [10]. Possible reason for this larger deviation could be the fact that the quark masses used in this calculation were fixed in units of temperature instead being tuned to give constant meson masses as lattice spacing is decreased. As discussed in Ref. [36] this could reduce the pressure by $10-15 \%$ at high temperatures. In Fig. 2 I also show the entropy density divided by the corresponding ideal gas value and compare the results of lattice calculations with resummed perturbative calculation $[32,33]$ as well as with the predictions from AdS/CFT correspondence for the strongly coupled regime [34]. The later is considerably below the lattice results. Note that pressure, energy density and the trace anomaly have also been recently discussed in the framework of resummed perturbative calculations which seem to agree with lattice data quite well [35].

The differences between the stout action and the $p 4$ and asqtad actions for the trace anomaly translates into the differences in the pressure and the energy density. In particular, the energy density is about $20 \%$ below the ideal gas limit for the stout action.
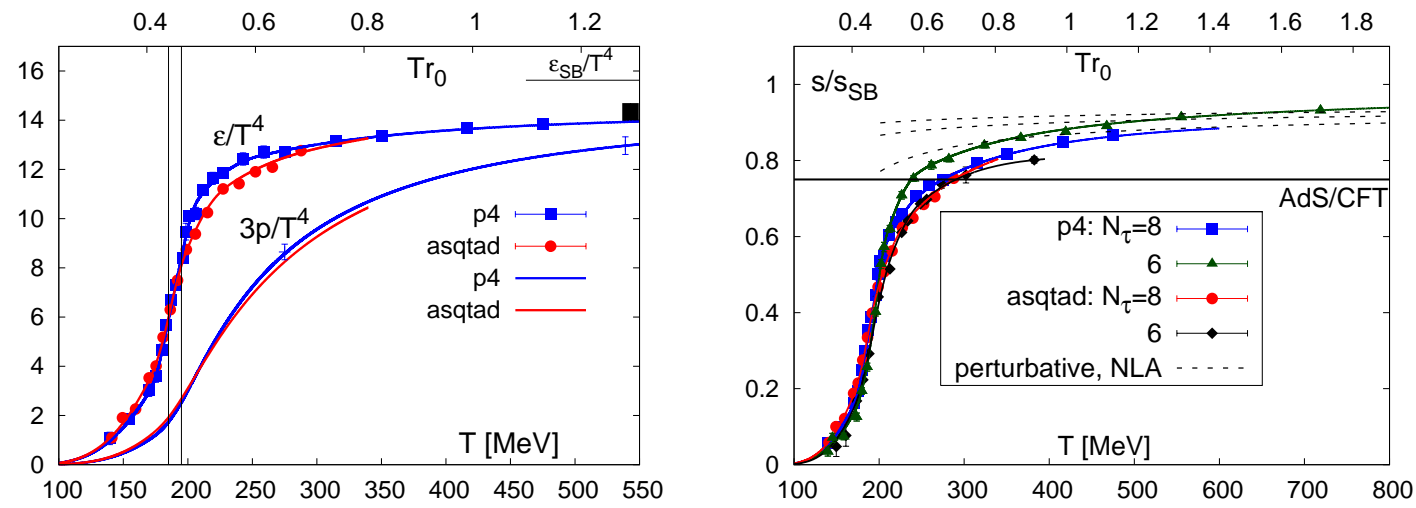

Figure 2: The energy density and the pressure as function of the temperature (left), and the entropy density divided by the corresponding ideal gas value (right). The dashed lines in the right panel correspond to the resummed perturbative calculations while the solid black line is the AdS/CFT result.

\section{Chiral and deconfinement transition}

The finite temperature transition in QCD has aspects related to deconfinement and chiral symmetry restoration. Deconfinement aspects of the transition are related to color screening and, for infinitely heavy quarks, also to the center symmetry. The order parameter for deconfinement is the Polyakov loop which is related to the free energy of isolated static quark. The breaking of the center symmetry is signaled by non-zero value of the Polyakov loop. In the opposite limit of massless quarks QCD has the chiral symmetry. The quark condensate $\langle\bar{\psi} \psi\rangle$ is the order parameter for this symmetry. The chiral symmetry is broken in the vacuum and expected to be restored at high temperatures. In Fig. 3 the renormalized Polyakov loop and the subtracted chiral condensate are shown. Both quantities show a smooth change in the transition region which is consistent with 

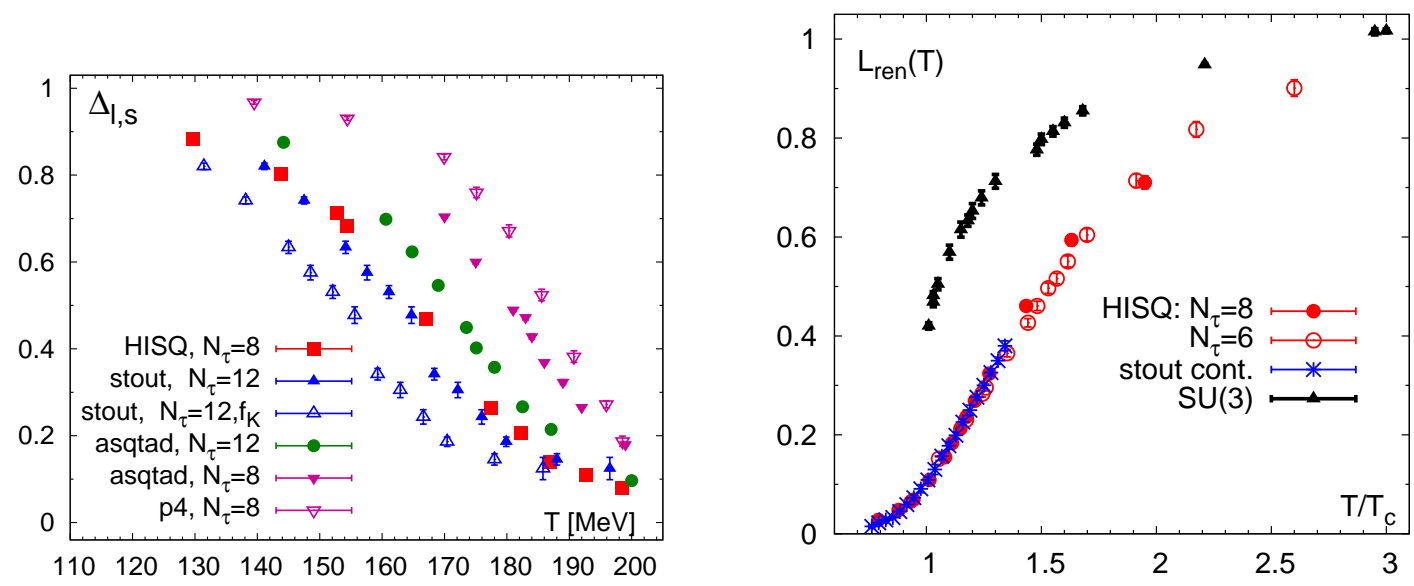

Figure 3: The subtracted chiral condensate (left) and the renormalized Polyakov loop (right) calculated for different actions. In the left panel we take $T_{c}=164 \mathrm{MeV}$ for the chiral temperature in QCD. The numerical data are taken from $[19,21,22,24,26,40]$.

the fact that the finite temperature transition is an analytic crossover and not a true phase transition [37]. The universal aspects of the chiral transition seem to be relevant for the range of quark masses which include the physical light quark mass and the corresponding transition temperature can be defined [38]. Calculations with the p4 action on $N_{\tau}=4$ and 6 lattices gave an estimate $T_{c}=192(4)(7) \mathrm{MeV}$ for the chiral transition temperature [15] which is significantly larger than the value of about $155 \mathrm{MeV}$ obtained for stout action ${ }^{1}$. This is due to large flavor symmetry breaking for the $\mathrm{p} 4$ action. As one can see from Fig. 3 the discrepancies between different actions are reduced when considering larger $N_{\tau}$ or the $H I S Q$ action where the effects of flavor symmetry breaking are much smaller. The preliminary estimate based on asqtad action for the chiral transition temperature based on asqtad action is $T_{c}=(164 \pm 6) \mathrm{MeV}$ [39]. The effects of flavor symmetry violations are smaller for the renormalized Polyakov loop. Only for temperatures $T<180 \mathrm{MeV}$ there are some discrepancies between the $\mathrm{p} 4$ and asqtad results obtained on $N_{\tau}=8$ lattices and the stout results. The asqtad results on $N_{\tau}=12$ lattices as well the HISQ results are in very good agreement [24, 26]. In Fig. 3 the renornalized Polyakov loop is shown for HISQ and stout actions as function of $T / T_{c}$. The results are compared to the renormalized Polyakov loop in pure gauge theory. Here $T_{c}$ denotes the phase transition temparature for pure gauge theory and the chiral transition temperature for QCD. As one can see except for very high temperatures the Polyakov loop in QCD is very different from the one in pure gauge theory and therefore it is not clear if the center symmetry plays an important role in QCD with light dynamical quarks.

\section{Correlation functions of static quark anti-quark pair}

One of the most prominent feature of the quark gluon plasma is the presence of chromoelectric (Debye) screening. The easiest way to study chromoelectric screening is to calculate the singlet

\footnotetext{
${ }^{1}$ Due to the crossover nature of the transition different chiral observable results in different estimate of the transition temperature, the so-called renormalized susceptibility calculated with stout action gives $T_{c} \simeq 147 \mathrm{MeV}$
} 

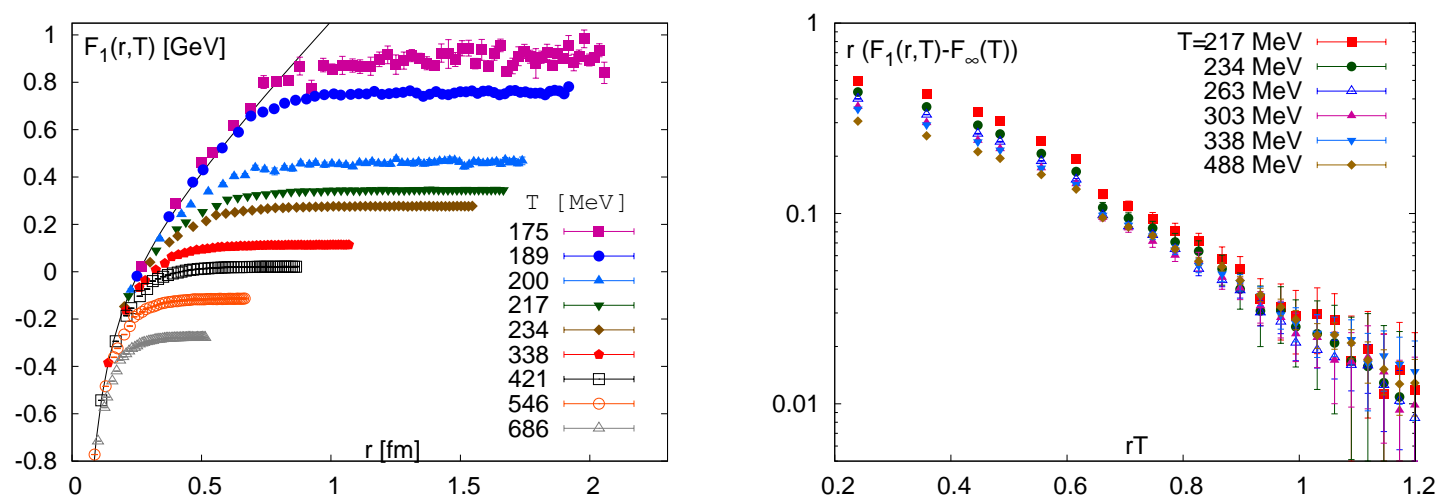

Figure 4: The singlet free energy $F_{1}(r, T)$ calculated in Coulomb gauge on $16^{3} \times 4$ lattices (left) and the combination $F_{1}(r, T)-F_{\infty}(T)$ as function of $r T$ (right). The solid black line is the parametrization of the zero temperature potential.

free energy of static quark anti-quark pair (for recent reviews on this see Ref. [41, 42]), which is expressed in terms of correlation function of temporal Wilson lines in Coulomb gauge

$$
\exp \left(-F_{1}(r, T) / T\right)=\frac{1}{N} \operatorname{Tr}\left\langle W(r) W^{\dagger}(0)\right\rangle
$$

$L=\operatorname{Tr} W$ is the Polyakov loop. The singlet free energy is in fact the logarithm of the static meson correlation function evaluated at $\tau=1 / T$. Instead of using the Coulomb gauge the singlet free energy can be defined in gauge invariant manner by inserting a spatial gauge connection between the two Wilson lines. Using such definition the singlet free energy has been calculated in $S U(2)$ gauge theory [43]. It has been found that the singlet free energy calculated this way is close to the result obtained in Coulomb gauge [43]. The singlet free energy turned out to be useful to study quarkonia binding at high temperatures in potential models (see e.g. Refs. [45, 46, 47, 48, 49]). The singlet free energy also appears naturally in the perturbative calculations of the Polyakov loop correlators at short distances [44].

The singlet free energy was recently calculated in QCD with one strange quark and two light quarks with masses corresponding to pion mass of $220 \mathrm{MeV}$ on $16^{3} \times 4$ lattices [50]. The numerical results are shown in Fig. 4. At short distances the singlet free energy is temperature independent and coincides with the zero temperature potential. In purely gluonic theory the free energy grows linearly with the separation between the heavy quark and anti-quark in the confined phase. In presence of dynamical quarks the free energy is saturated at some finite value at distances of about $1 \mathrm{fm}$ due to string breaking $[41,51,53]$. This is also seen in Fig. 4. Above the deconfinement temperature the singlet free energy is exponentially screened at sufficiently large distances $[40,52]$ with the screening mass proportional to the temperature, i.e.

$$
F_{1}(r, T)=F_{\infty}(T)-\frac{4}{3} \frac{g^{2}(T)}{4 \pi r} \exp \left(-m_{D}(T) r\right), m_{D} \sim T
$$

Therefore in Fig. 4 we also show the combination $F_{1}(r, T)-F_{\infty}(T)$ as a function of $r T$. As one can see from the figure this function shows an exponential fall-off at distances $r T>0.8$. The fact that the slope is the same for all temperatures means that $m_{D} \sim T$, as expected. 


\section{Heavy and light meson correlation functions}

Information on hadron properties at finite temperature as well as the transport coefficients are encoded in different spectral functions. In particular the fate of different quarkonium states in QGP can studied by calculating the corresponding quarkonium spectral functions (for a recent review see Ref. [42]). On the lattice we can calculate correlation function in Euclidean time. This is related to the spectral function via integral relation

$$
G(\tau, T)=\int_{0}^{\infty} d \omega \sigma(\omega, T) K(\tau, \omega, T), K(\tau, \omega, T)=\frac{\cosh (\omega(\tau-1 / 2 T))}{\sinh (\omega / 2 T)}
$$

Given the data on the Euclidean meson correlator $G(\tau, T)$ the meson spectral function can be calculated using the Maximum Entropy Method (MEM) [54]. For charmonium this was done by using correlators calculated on isotropic lattices $[55,56]$ as well as anisotropic lattices $[57,58$, 59] in the quenched approximation. It has been found that quarkonium correlation function in Euclidean time show only very small temperature dependence [56, 59]. In other channels, namely the vector, scalar and axial-vector channels stronger temperature dependence was found $[56,59]$. The spectral functions in the pseudo-scalar and vector channels reconstructed from MEM show peak structures which may be interpreted as a ground state peak $[56,57,58]$. Together with the weak temperature dependence of the correlation functions this was taken as strong indication that the $1 \mathrm{~S}$ charmonia $\left(\eta_{c}\right.$ and $\left.J / \psi\right)$ survive in the deconfined phase to temperatures as high as $1.6 T_{c}$ $[56,57,58]$. A detailed study of the systematic effects show, however, that the reconstruction of the charmonium spectral function is not reliable at high temperatures [59], in particular the presence of peaks corresponding to bound states cannot be reliably established. Presence of large cutoff effects at high frequencies also complicates the analysis [62]. The only statement that can be made is that the spectral function does not show significant changes within the errors of the calculations. Recently quarkonium spectral functions have been studied using potential models and lattice data for the singlet free energy of static quark anti-quark pair [47, 48, 49]. These calculations show that all charmonium states are dissolved at temperatures smaller than $1.2 T_{c}$, but the Euclidean correlators do not show significant changes and are in fairly good agreement with available lattice data both for charmonium [56,59] and bottomonium [59, 60]. This is due to the fact that even in absence of bound states quarkonium spectral functions show significant enhancement in the threshold region [46]. Therefore previous statements about quarkonia survival at high temperatures have to be revisited. Exploratory calculations of the charmonium correlators and spectral functions in 2-flavor QCD have been reported in Ref. [61] and the qualitative behavior of the correlation functions was found to be similar.

The large enhancement of the quarkonium correlators above deconfinement in the scalar and axial-vector channel can be understood in terms of the zero mode contribution $[46,63]$ and not due to the dissolution of the $1 P$ states as previously thought. Similar, though smaller in magnitude, enhancement of quarkonium correlators due to zero mode is seen also in the vector channel [59]. Here it is related to heavy quark transport [45, 64]. Due to the heavy quark mass the Euclidean correlators for heavy quarkonium can be decomposed into a high and low energy part $G(\tau, T)=G_{\text {low }}(\tau, T)+G_{\text {high }}(\tau, T)$ The area under the peak in the spectral functions at zero energy $\omega \simeq 0$ giving the zero mode contribution to the Euclidean correlator is proportional to some 
susceptibility, $G_{\text {low }}^{i}(\tau, T) \simeq T \chi^{i}(T)$, which have been calculated on the lattice in Ref. [65]. It is natural to ask whether the generalized susceptibilities can be described by a quasi-particle model. The generalized susceptibilities have been calculated in Ref. [66] in the free theory. Replacing the bare quark mass entering in the expression of the generalized susceptibilities by an effective temperature dependent masses one can describe the zero mode contribution very well in all channels [65].

While temporal correlators are not sensitive to the change in the spectral functions spatial quarkonium correlation functions could be more sensitive to this. Recent lattice calculations show indication for significant change in spatial charmonium correlators above deconfinement[67].

The spectral function for light mesons has been calculated on the lattice in quenched approximation $[68,69,70,71]$. However, unlike in the quarkonia case the systematic errors in these calculations are not well understood. The spatial meson correlation function provide interesting insight into the modification of the spectral functions also in the light quark sector. In particular, the study of the spatial meson correlation function in the vector and axial vector channel indicate the degeneracy of the vector and axial vector spectral functions at the chiral transition [72]. At the same time the lattice calculations of the pseudo-scalar and scalar correlation function indicate that the $U_{A}(1)$ axial symmetry is only restored at temperatures significantly higher than the chiral transition temperature [72].

\section{Conclusions}

In recent years significant progress has been achieved in studying strongly interacting matter at high temperatures using lattice QCD. Equation of state and transition temperature have been calculated at several lattice spacings allowing for controlled continuum extrapolations. Current lattice data suggest a transition temperature for chiral symmetry restoration of $(147-164) \mathrm{MeV}$. Temporal meson correlation functions have been also studied in lattice QCD but the numerical data are not precise enough to provide detailed information of the corresponding meson spectral functions. However, these lattice data are useful to constrain the model calculations of the meson spectral functions. Spatial meson correlators provide additional information about meson spectral functions, in particular indication of effective restoration of the $U_{A}(1)$ symmetry at temperatures larger than the chiral transition temperature.

\section{References}

[1] D. Gross, R. Pisarski, L. Yaffe, Rev. Mod. Phys. 53, 43 (1981)

[2] B. Müller and J. Nagle, Ann. Rev. Nucl. Part. Sci. 56, 93 (2006)

[3] U. Wiedemann, Nucl. Phys. A, 830, 74c (2009)

[4] J. Kuti, J. Polónyi and K. Szlachányi, Phys. Lett. B 98 (1981) 199;

[5] L. D. McLerran and B. Svetitsky, Phys. Rev. D 24 (1981) 450;

[6] J. Engels, F. Karsch, H. Satz and I. Montvay, Phys. Lett. B 101 (1981) 89.

[7] G. Boyd et al., Nucl. Phys. B 469, 419 (1996) 
[8] C. W. Bernard et al., [MILC Collaboration], Phys. Rev. D 55 (1997) 6861

[9] A. Ali Khan et al., [CP-PACS Collaboration], Phys. Rev. D 64 (2001) 074510

[10] F. Karsch, E. Laermann and A. Peikert, Phys. Lett. B 478 (2000) 447;

[11] P. Petreczky, Nucl. Phys. Proc. Suppl. 140, 78 (2005);

[12] E. Laermann and O. Philipsen, Ann. Rev. Nucl. Part. Sci. 53, 163 (2003)

[13] C. Bernard et al. [MILC Collaboration], Phys. Rev. D 71, 034504 (2005)

[14] Y. Aoki, Z. Fodor, S. D. Katz and K. K. Szabo, JHEP 0601, 089 (2006)

[15] M. Cheng et al., Phys. Rev. D 74, 054507 (2006)

[16] Y. Aoki, Z. Fodor, S. D. Katz and K. K. Szabo, Phys. Lett. B 643 (2006) 46 [arXiv:hep-lat/0609068].

[17] C. Bernard et al., Phys. Rev. D 75 (2007) 094505

[18] M. Cheng et al., Phys. Rev. D 77 (2008) 014511

[19] M. Cheng et al., Phys. Rev. D 81, 054504 (2010)

[20] A. Bazavov et al., Phys. Rev. D 80 (2009), 014504

[21] Y. Aoki, et al, JHEP 0906 (2009) 088

[22] S. Borsanyi et al., [Wuppertal-Budapest Collaboration], arXiv:1007.2580 [hep-lat].

[23] S. Borsanyi, et al., [Wuppertal-Budapest Collaboration], arXiv:1005.3508 [hep-lat].

[24] A. Bazavov and P. Petreczky [HotQCD collaboration], J. Phys. Conf. Ser. 230, 012014 (2010);

[25] A. Bazavov and P. Petreczky [HotQCD Collaboration], PoS LAT2009, 163 (2009)

[26] A. Bazavov, P. Petreczky, arXiv:1009.4914v1 [hep-lat]

[27] P. Petreczky, Nucl. Phys. A 830, 11C (2009)

[28] C. DeTar and U. M. Heller, Eur. Phys. J. A 41, 405 (2009);

[29] Z. Fodor and S. D. Katz, arXiv:0908.3341 [hep-ph];

[30] K. Orginos, D. Toussaint and R. L. Sugar [MILC Collaboration], Phys. Rev. D 60 (1999) 054503

[31] P. Huovinen and P. Petreczky, Nucl. Phys. A 837, 26 (2010)

[32] J. P. Blaizot, E. Iancu and A. Rebhan, Phys. Rev. Lett. 83 (1999) 2906;

[33] Phys. Rev. D 63 (2001) 065003

[34] S.S. Gubser, I.R. Klebanov and I.I Tseytlin, Nucl. Phys. B 534 (1998) 202

[35] J. O. Andersen, L. E. Leganger, M. Strickland and N. Su, arXiv:1009.4644 [hep-ph].

[36] F. Csikor, et al., JHEP 0405 (2004) 046

[37] Y. Aoki, et al., Nature 443, 675 (2006)

[38] S. Ejiri, et al., Phys. Rev. D80, 094505 (2009). [arXiv:0909.5122 [hep-lat]].

[39] W. Soeldner (HotQCD Coll.), talk given at Lattice 2010, to be published in the proceedings

[40] O. Kaczmarek et al., Phys. Lett. B 543, 41 (2002)

[41] P. Petreczky, Eur. Phys. J. C 43, 51 (2005) 
[42] A. Bazavov, P. Petreczky, A. Velytsky, [arXiv:0904.1748 [hep-ph]].

[43] A. Bazavov, P. Petreczky and A. Velytsky, Phys. Rev. D 78, 114026 (2008)

[44] N. Brambilla, et. al., arXiv:1007.5172 [hep-ph].

[45] Á. Mócsy and P. Petreczky, Eur. Phys. J. C 43, 77 (2005);

[46] Á. Mócsy and P. Petreczky, Phys. Rev. D 73, 074007 (2006)

[47] Á. Mócsy and P. Petreczky, Phys. Rev. Lett. 99, 211602 (2007);

[48] Á. Mócsy and P. Petreczky, Phys. Rev. D 77, 014501 (2008);

[49] Á. Mócsy and P. Petreczky, Eur. Phys. J. ST 155, 101 (2008)

[50] RBC-Bielefeld Collaboration, work in progress

[51] P. Petreczky and K. Petrov, Phys. Rev. D 70, 054503 (2004)

[52] S. Digal, S. Fortunato and P. Petreczky, Phys. Rev. D 68, 034008 (2003)

[53] O. Kaczmarek and F. Zantow, Phys. Rev. D 71, 114510 (2005)

[54] M. Asakawa, T. Hatsuda and Y. Nakahara, Prog. Part. Nucl. Phys. 46, 459 (2001)

[55] S. Datta et al., Nucl. Phys. Proc. Suppl. 119, 487 (2003)

[56] S. Datta, et al., Phys. Rev. D 69, 094507 (2004)

[57] T. Umeda, K. Nomura and H. Matsufuru, Eur. Phys. J. C 39S1, 9 (2005)

[58] M. Asakawa and T. Hatsuda, Phys. Rev. Lett. 92, 012001 (2004)

[59] A. Jakovác et al., Phys. Rev. D 75, 014506 (2007)

[60] S. Datta, et al., AIP Conf. Proc. 842, 35 (2006)

[61] G. Aarts, C. Allton, M. B. Oktay, M. Peardon and J. I. Skullerud, Phys. Rev. D 76, 094513 (2007)

[62] F. Karsch, et al., Phys. Rev. D 68, 014504 (2003) [arXiv:hep-lat/0303017].

[63] T. Umeda, Phys. Rev. D 75, 094502 (2007)

[64] P. Petreczky and D. Teaney, Phys. Rev. D 73, 014508 (2006)

[65] P. Petreczky, Eur. Phys. J. C 62, 85 (2009)

[66] G. Aarts and J. M. Martinez Resco, Nucl. Phys. B 726 (2005) 93

[67] S. Mukherjee, Nucl. Phys. A 820 (2009) 283C

[68] F. Karsch et al., Phys. Lett. B 530, 147 (2002);

[69] F. Karsch et al., Nucl. Phys. A 715, 701 (2003)

[70] M. Asakawa, T. Hatsuda and Y. Nakahara, Nucl. Phys. A 715, 863 (2003)

[71] G. Aarts, et al., Phys. Rev. Lett. 99, 022002 (2007)

[72] M. Cheng, et al., [arXiv:1010.1216 [hep-lat]]. 